REVIEW

\title{
The impact of hormone physiology on clinical practice with reference to two papers* spanning 40 years of endocrinology
}

\section{P C Hindmarsh}

Arch Dis Child 2005;90:1144-1147. doi: 10.1136/adc.2004.069682

Following the pioneering work of Roger Ekins in describing the methodology for radioimmunoassay of hormone concentrations in plasma a series of publications followed describing variation in plasma hormone concentrations in a number of diseases. Though fluctuations in hormonal concentrations had been suspected, it was not until Hunter and Rigal documented these for the GH axis that it became apparent that the preferred mode of signalling for many hormone systems and in particular the anterior-pituitary hormones was of a pulsatile nature.

Correspondence to: Prof. P C Hindmarsh Cobbold Laboratories, Middlesex Hospital, Mortimer Street, London WIT 3AA, UK; p.hindmarsh@úcl.ac.uk

Accepted 9 May 2005
$\mathrm{T}$ he observation that many of the hormone systems operated in a pulsatile mode had profound implications for the manner in which testing for hormone excess or deficiency was undertaken. Furthermore, the observations reinforced in the minds of endocrinologists the need to tailor hormone replacement to mimic as near as possible physiological secretion. Hunter and Rigal's paper ${ }^{1}$ is as applicable to our practice some 40 years later as it was to the endocrinologist unravelling the complex nature of disease processes and therapeutic options in the late 1960s.

\section{DIAGNOSTIC IMPLICATIONS OF HORMONE PULSATILITY}

Analysis of hormones in the circulation forms the backbone of endocrine practice. Such measures may use single samples or be derived from in hormone secretion take place on a day to day basis. Variations are not confined to short time intervals, as evidenced by the changes in gonadotrophins during the menstrual cycle. The majority of tests conducted in paediatric practice take place at a fixed time of the day. In the execution and interpretation of these tests, consideration needs to be given to the physiology of the hormones studied and the sampling interval chosen for the collection of blood samples.

Many of the analytes measured in clinical endocrine practice do not have a circadian rhythm or are complexed in the serum to binding proteins. In such situations, it can be assumed that there are not long term variations, although this may not always be the case (table 1 ). Cortisol, the gonadotrophins, growth hormone $(\mathrm{GH})$, and insulin are the best known of the the measurement of multiple samples. Variations hormones that pulse. $^{2-4}$ The implication of pulsatile secretion assumes great importance in assessing the performance of diagnostic tests. The efficiency, sensitivity, and specificity of these tests will all be dependent to some extent on the repeatability and reproducibility of the test under study and the pulsatile system will influence both of these in a number of ways.

Most endocrine tests are conducted over short periods of time and their results are often extrapolated to a longer time frame. For example, GH provocation tests used to assess the $\mathrm{GH}$ secretory state in children are performed over a two hour period, and the results are then compared with height velocity measurements obtained over a longer period of time, often one year. That there is a relationship is perhaps surprising; that there are false-positive and falsenegative results, perhaps not. Hormone pulsatility may also influence diagnostic tests, if the test itself (for example, stimulus applied) is influenced by oscillation of the system under study.

Following the observations of Hunter and Rigal it became clear that the oscillatory system that led to the generation of $\mathrm{GH}$ pulses also appeared to influence the $\mathrm{GH}$ response to a particular stimulus such as insulin induced hypoglycaemia. If the serum GH concentration at the start of the study was measurable, then the rise in $\mathrm{GH}$ in response to the hypoglycaemic stimulus was likely to be attenuated. ${ }^{5}$

Understanding hormone physiology is therefore essential, both in defining the circadian rhythm in the variability of the hormone concentration under study and also in beginning to understand from the pattern of secretion what the underlying regulatory processes are. The realisation that the $\mathrm{GH}$ pulse arose as a result of a balance between the actions of two hypothalamic peptides, GH-releasing hormone and somatostatin, as well as a third factor produced in the stomach, ghrelin, greatly facilitated the interpretation of $\mathrm{GH}$ testing. A pulse of $\mathrm{GH}$ appears to arise as a result of a coordinated decrease in somatostatin secretion, which is inhibitory to GH-release, coupled with an increase in GH-releasing hormone secretion

*Hunter WM, Rigal WM. Diurnal pattern of plasma growth hormone concentration in children and adolescents. J Endocrinol 1966;34:147-53.

Doyle EA, Weinzimer SA, Steffen AT, et al. A randomized, prospective trial comparing the efficacy of continuous subcutaneous insulin infusion with multiple daily injections using insulin glargine. Diabetes Care 2004;27:1554-8. 
Table 1 Within-individual coefficients of variation for several hormones (data derived from Saini et a ${ }^{10}$ )

Coefficient of variation (\%) Mean (range)

Mean $24 \mathrm{~h}$ serum $\mathrm{GH}$ conc. $35(9-58)$ Serum IGF-1 conc Serum testosterone conc $21(14-34)$ $13(8-19)$

which is stimulatory. These two factors either cycle out of phase with each other or else the GH-releasing hormone exposure is constant and only somatostatin oscillates. ${ }^{67}$ Figure 1 shows the effect of differing states of GH secretion before the application of the stimulus (GH-releasing hormone) and how the response to the same exogenous stimulus varies.

With time it has become apparent that other hormones/ environmental factors also influence the amount of GH that might be released at any point in time. Thyroxine and cortisol, which directly alter gene transcription, will influence the results obtained and these need to be normalised before undertaking a diagnostic study. Similarly, the presence of high plasma glucose concentrations or free fatty acid concentrations will influence the response obtained. Alterations of sex steroid concentrations during puberty (or even during the course of the menstrual cycle) modify the GH response to GH-releasing hormone.

Endocrine systems are also subject to feedback from target tissues; this becomes an issue not only in the interpretation of single provocation tests, but also where second tests are performed in rapid succession to the first. This is well documented in the GH field, where a diminished response to a second stimulus of GH-releasing hormone applied 1, 2, or 3 hours after the first can be observed. ${ }^{8}$ A gradual diminution in responsitivity of the anterior pituitary can be documented with this type of approach. The practical implications of this in undertaking two tests in the same day, often following each other, are immense because the cut-off that might be applied to determine normality or abnormality may not be the same for the second test as for the first, especially if the second stimulus is different from the first.

In assessing the results of endocrine evaluations, it is generally assumed that the single or multiple samples measured are relatively stable, at least over short periods. This is unlikely in biological systems and in the methods used to measure the hormone of interest. In endocrine practice, we are well aware of the errors incurred in the measurement of hormones, and the between and within assay coefficients of variation aid greatly the interpretation of these hormone measurements. In the short term, a number of studies have shown variability within and between individuals in terms of endocrine tests. ${ }^{9-11}$ Group data are usually reproducible, but problems can arise if it is assumed that individual oscillatory profiles are consistent from day to day (table 1). These observations add an additional dimension to the comparison of studies obtained under one series of circumstances with a set obtained under another series of circumstances, particularly if they are separated over long periods of time.

\section{THERAPEUTIC IMPLICATIONS OF HORMONE PULSATILITY}

Hunter and Rigal's observations reinforced general feeling at the time that endocrine replacement therapy should approximate as closely as is possible the physiological pattern of hormone production. At that time GH hormone therapy was in its infancy and supplies of pituitary derived GH were in short supply. As a consequence GH was usually administered

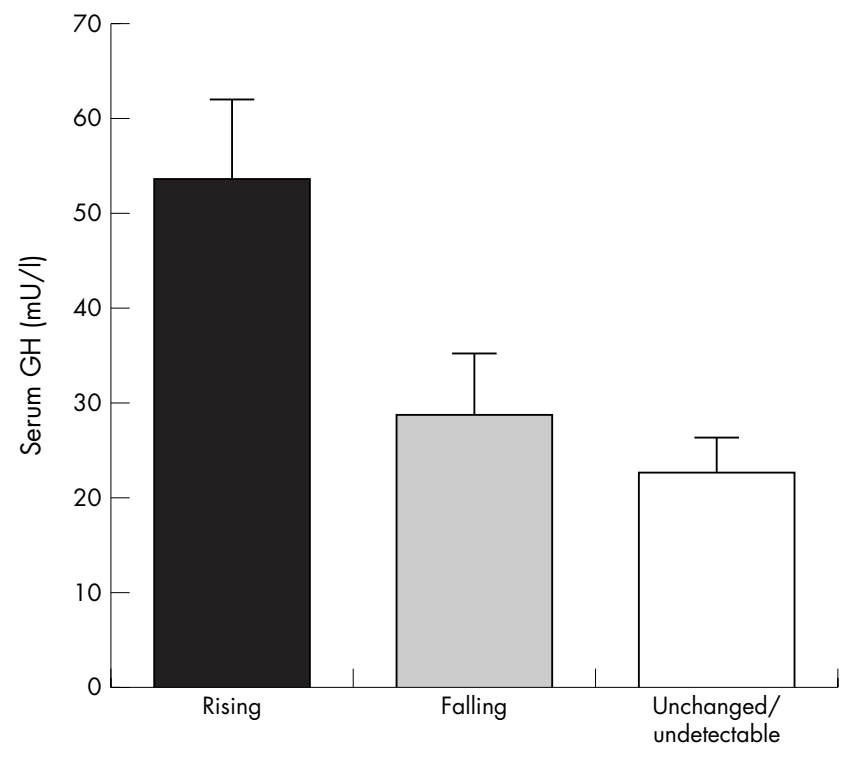

Figure 1 Effect of pre-stimulus serum GH concentration on the subsequent serum $\mathrm{GH}$ response to $\mathrm{GH}$-releasing hormone.

two to three times per week, a pattern which could broadly be considered to reflect the single nocturnal pulse of $\mathrm{GH}$ observed in the physiological situation (fig 2). The situation generated quite good growth acceleration, but over time daily injections were shown to be superior. As time progressed it became apparent that the components of the pulsatile system, namely the amplitude of the peaks and the trough concentrations, also played an important role in determining the metabolic impact of GH. ${ }^{12}$

Given these physiological observations the question is often posed as to how does GH treatment by once daily subcutaneous injection work. Growth rate is largely determined by the amplitude of the GH secretory peak, which is analogous to the concentration achieved after subcutaneous injection. The pharmacokinetics of subcutaneous GH are such that the GH is cleared completely from the circulation 12 hours after administration. Hence the physiological situation is maintained to a degree with a mimicked secretory burst followed by a period of no secretion. What is perhaps more in question is how much does the tail of the subcutaneous profile contribute, and whether there could be any adverse effect of such exposure over the 12 hour period.

Despite the wide range of medications used by clinical endocrinologists, the principles derived from analysis of hormone pulsatility have not been widely applied in therapeutic practice. With respect to insulin treatment the beneficial effect in terms of maintenance of normoglycaemia comes from tailoring insulin to food intake and not the other way round. Pulsatile insulin administration is highly effective at regulating blood glucose concentrations as it avoids downregulation of the insulin receptor. This observation has been applied in the clinical setting for the management of diabetic ketoacidosis where a continuous intravenous insulin infusion is utilised to produce a graded reduction in the blood glucose concentration.

Despite the documentation of insulin pulsatility from the late 1970s onwards, it is only more recently that it has become possible to deliver insulin in a manner that mimics physiology. This has arisen from the advances in biotechnology leading to the generation of ultra short acting and long acting insulins (table 2) ${ }^{13}$ and better methodologies for matching insulin delivery to food (carbohydrate intake). 


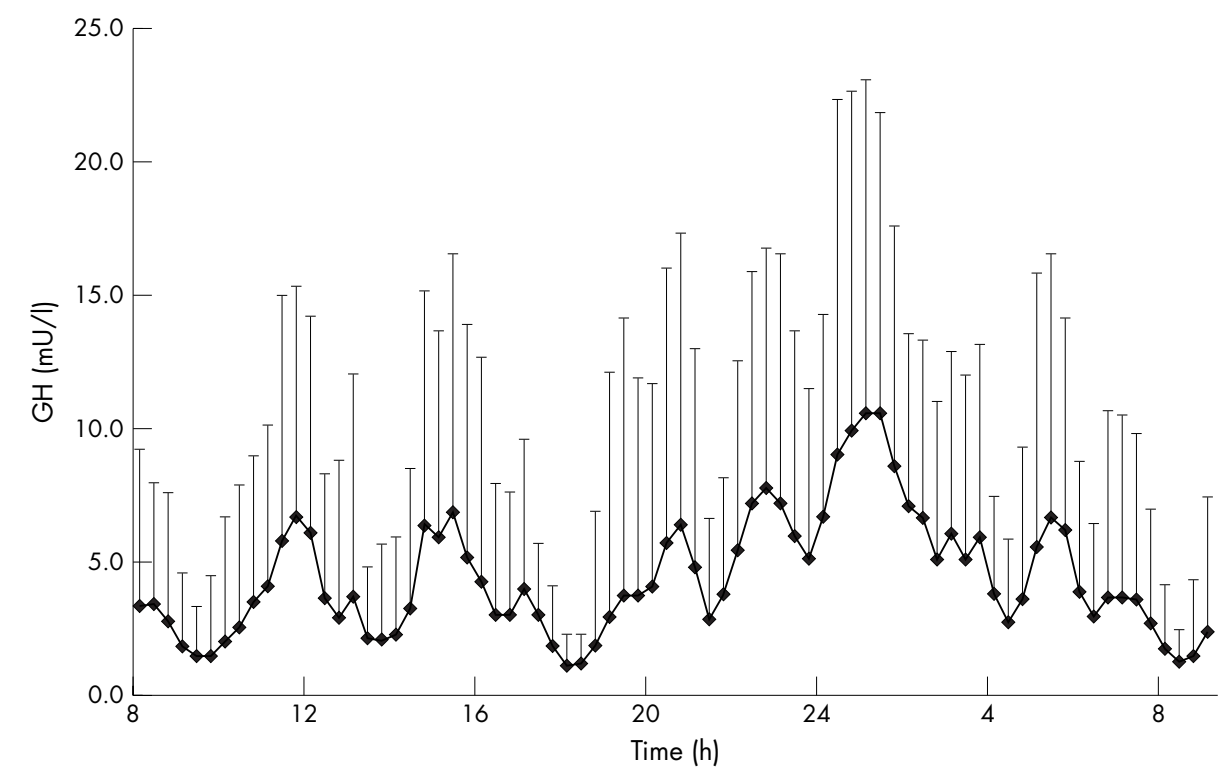

Figure 2 Twenty four hour serum GH concentration profile from 29 children.

Figure 3 shows how insulin concentrations might vary over a 24 hour period. At least two components can be identified: first, the excursions associated with meals; and second, a background basal secretory rate. Within both are additional oscillations of insulin occurring on an approximately 13 minute cycle. ${ }^{414}$ One of the problems with devising insulin delivery systems has been the types of insulin available for injection. Until the advent of the ultra short acting insulin analogues along with more square wave forms of continuous insulin delivery such as glargine or detemir, it had not be possible to get away from the need to cover unexpected periods of insulin exposure with snacks and to ensure that there was rigorous timing of meals. Multiple dosing insulin regimens using these modern insulin analogues now allow clinicians to devise more physiological insulin delivery to match day to day activities and should also reduce overall insulin exposure which may in itself be problematic. This allows the person with diabetes a more flexible lifestyle as it obviates the need for fixed meal times and associated snacks and opens the way for easier adjustments in the face of exercise and other activities. ${ }^{15}$ In theory it allows individuals almost a free range with diet because insulin delivery is tailored to food intake and not the other way round. Like any other individual who does not have diabetes, however, the consequences of a more liberal regimen are that if food intake is in excess, weight gain and obesity will result.

For optimal control the long acting component needs to constitute about $30-50 \%$ of total of the daily dose and the ultra short acting boluses that accompany food intake need to be matched to the carbohydrate content (and/or glycaemic index) of the food ingested. Additional improvements in glycaemic control can be achieved using this multiple dose insulin regimen and further steps to improve the insulin delivery using continuous subcutaneous insulin infusion devices (insulin pumps) can lead to further improvement in glycosylated haemoglobin concentration. ${ }^{16}$ The appliance of our understanding of hormone physiology, in this case insulin, can lead to near normal blood glucose concentrations. The result of this is that considerable interest has centred on closing the loop so that automated sensing of tissue glucose concentrations can be utilised as the major determinant of the regulation of insulin delivery using these pump technologies. These advances should be welcomed by all, despite their seeming complexity, as approaching the ideal that we all strive towards, namely the approximation of hormone replacement to as near a physiological standard as is possible.

\section{CONCLUSION}

Pulsatility is a feature of endocrine systems and needs to be considered in the analysis of information derived from physiological and pathophysiological situations. To me the main point of Hunter and Rigal's paper was that careful consideration needs to be given to understanding the physiology and pathophysiology of the conditions we face in day to day paediatric practice. Laboratory investigations are an important component of the diagnostic process, so a close understanding of the environment in which the test is conducted is required. By this is meant not simply the factors that we know might alter the production of the analyte under investigation, but also the regulatory systems which are involved in determining the circulating concentration and the mechanisms by which the substance is cleared from the circulation. In the GH axis the practical implications of these

\begin{tabular}{|llll}
\hline \multicolumn{4}{l}{ Table 2 Pharmacodynamics of insulin and insulin analogues } \\
\hline Insulin type & Onset (h) & Peak (h) & Duration (h) \\
\hline $\begin{array}{llll}\text { Insulin analogues } \\
\quad \text { Rapid acting (e.g. Novorapid or Humalog) }\end{array}$ & 0.25 & 1 & $3-4$ \\
$\quad \begin{array}{l}\text { Long acting (e.g. glargine or detemir) } \\
\text { Standard insulins } \\
\quad \text { Regular }\end{array}$ & $2-3$ & None & $20-24$ \\
$\quad$ Insulatard & $0.5-1$ & $2-3$ & $3-8$ \\
\hline & $3-4$ & $4-12$ & $10-20$ \\
\hline
\end{tabular}




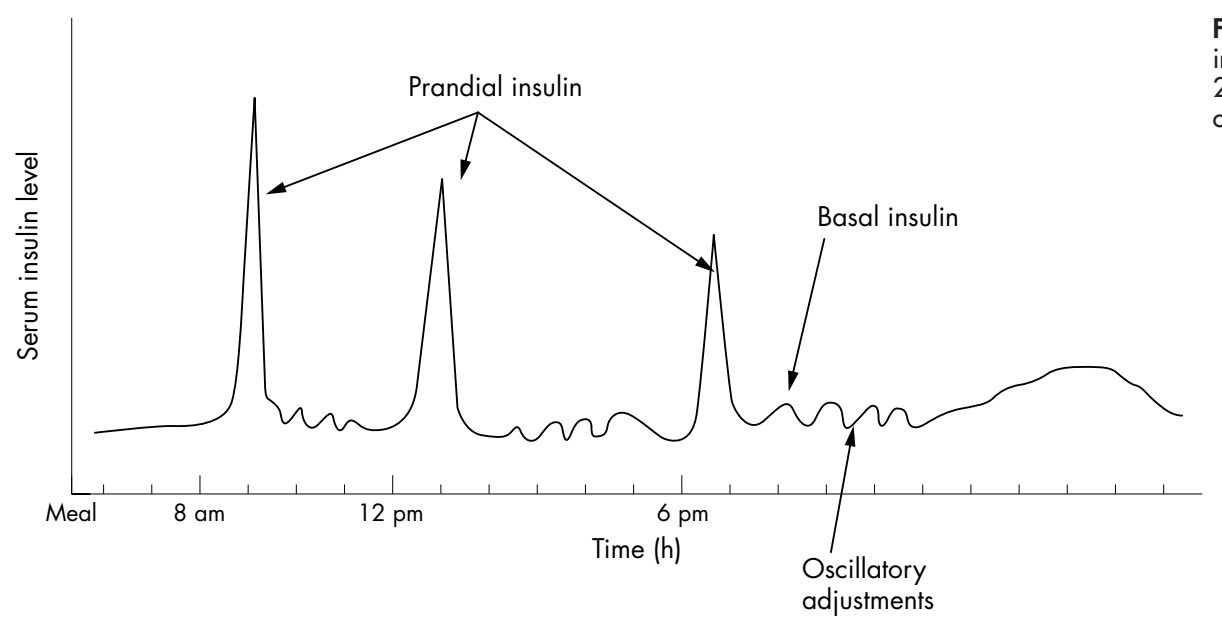

Figure 3 Representation of variation in serum insulin concentration during a 24 hour period to illustrate components of insulin secretion/delivery.

observations are that cortisol, thyroxine, and sex steroid status should be known before undertaking GH testing. The test should be undertaken using a known stimulus such as glucagons; 2-3 blood samples should be drawn over a 30 minute period prior to the injection of the stimulus in order to determine whether serum GH concentrations are unchanged, rising, or falling, so that an adequate interpretation of the subsequent $\mathrm{GH}$ response to the stimulus can be made. Hunter and Rigal's data have allowed us to undertake more detailed studies of hormone secretion over longer periods of time, and have allowed us to realise that individual variation can be observed and needs to be taken into account in the assessment of the likelihood of disease presence or absence.

There are increasing examples in the literature where the physiological delivery of a hormone has been shown to be the most effective mode for replacement therapy. Hormone pulsatility studies of insulin secretion tell us that the multiple dose insulin regimen is likely to be the most efficient and efficacious in terms of total dose administered, glycaemia achieved, and long term complications attenuated. This system can be further refined using programmable insulin pumps to achieve normal glycaemia.

Competing interests: $\mathrm{PCH}$ consults on Insulin Pump therapy to Medtronic UK

\section{REFERENCES}

1 Hunter WM, Rigal WM. Diurnal pattern of plasma growth hormone concentration in children and adolescents. J Endocrinol 1966;34:147-53.

2 Krieger DT. Rhythms in CRF, ACTH and corticosteroids. In: Krieger DT, ed. Endocrine rhythms. New York: Raven Press, 1979.
3 Clayton RN, Royston JP, Chapman J, et al. Is changing hypothalamic activity important for control of ovulation? BMJ 1987;295:7-12.

4 Goodner CJ, Walike BC, Koerker DJ, et al. Insulin, glucogen and glucose exhibit synchronous sustained oscillations in fasting monkeys. Science 1977; 195: 177-9

5 Youlton R, Kaplan SL, Grumbach MM. Growth and growth hormone. IV limitations of the growth hormone response to insulin and arginine in the assessment of growth hormone deficiency in children. Pediatrics 1969;43:989-1004.

6 Hindmarsh PC, Brain CE, Robinson ICAF, et al. The interaction of growth hormone releasing hormone and somatostatin in the generation of $\mathrm{GH}$ pulse in man. Clin Endocrinol 1991:35:353-60.

7 Achermann JC, Hindmarsh PC, Robinson ICAF, et al. The relative roles of $\left(\mathrm{GHRH}(1-29) \mathrm{NH}_{2}\right)$ and intermittent somatostatin (1-14)(SS) in growth hormone (GH) pulse generation: studies in normal and post cranial irradiated individuals. Clin Endocrinol 1999;51:587-95.

8 Suri D, Hindmarsh PC, Matthews DR, et al. The pituitary gland is capable of responding to two successive doses of growth hormone releasing hormone (GHRH). Clin Endocrinol 1991;34:13-17.

9 Donaldson DL, Holowell JG, Pan F, et al. Growth hormone secretion profiles: variation on consecutive nights. J Pediatr 1989;115:51-6.

10 Saini S, Hindmarsh PC, Matthews DR, et al. Reproducibility of 24 hour serum growth hormone profiles in man. Clin Endocrinol 1991;34:455-62.

11 Bridges NA, Hindmarsh PC, Pringle PJ, et al. Cortisol, androsteredine, dehydroepidrosterone sulphate and 17 hydroxyprogesterone responses to low dose of (1-24)ACTH. J Clin Endocrinol Metab 1998;83:3750-3.

12 Hindmarsh PC, Dennison E, Pincus SM, et al. A sexually dimorphic pattern of growth hormone secretion in the elderly. J Clin Endocrinol Metab 1999:84:2679-85.

13 Hirsch IB. Insulin analogues. N Engl J Med 2005;352:174-83.

14 Lang DA, Matthews DR, Peto J, et al. Cyclic oscillations of basal plasma glucose and insulin concentrations in human beings. N Engl J Med 1979:301:1023-7.

15 Tuominen JA, Karonen S-L, Melamies L, et al. Exercise induced hypoglycaemia in IDDM patients treated with a short acting insulin analogue. Diabetologia 1995;38:106-11

16 Doyle EA, Weinzimer SA, Steffen AT, et al. A randomized, prospective trial comparing the efficacy of continuous subcutaneous insulin infusion with multiple daily injections using insulin glargine. Diabetes Care 2004;27:1554-8. 